\title{
Peripartum Ultrasound-Guided Drainage of Cystic Fetal Sacrococcygeal Teratoma for the Prevention of the Labor Dystocia: A Report of Two Cases
}

\author{
Vedran Stefanovic, M.D., Ph.D., ${ }^{1}$ and Erja Halmesmäki, M.D., Ph.D. ${ }^{1}$
}

Fetal sacrococcygeal teratoma (SCT) is the most common tumor in the neonatal period and is easily detected by modern ultrasound techniques, mostly during the secondtrimester screening. It can cause significant fetal/neonatal morbidity and mortality due to its size, vascular loading, possible rupture, and labor dystocia. Mostly cystic teratomas have favorable prognosis, but if untreated in utero, they may rupture or cause labor obstruction. Cesarean delivery, especially with the vertical incision, increases significantly maternal morbidity due to the hemorrhage and the risk of the uterine rupture in the subsequent pregnancies. The authors report in details two SCT cases with uncomplicated vaginal delivery after peripartum ultrasound-guided drainage of the cystic teratoma. We conclude that the percutaneous emptying of the cystic SCT is an easy, encouraging, safe, and efficient procedure and enables normal vaginal delivery, thus avoiding labor dystocia and possible complications of the cesarean delivery and the risk of tumor rupture.

KEYWORDS: Fetal sacrococcygeal teratoma, prenatal diagnosis, fetal surgery, prevention of dystocia, ultrasound

Fetal sacrococcygeal teratoma (SCT) is seen in 1 in every 35,000 live births, and it is the most common tumor presenting in newborn humans. ${ }^{1,2}$ This usually benign fetal tumor develops at the base of the tailbone. $\mathrm{SCT}$ s derive from the pluripotent cell line that escapes from the control of embryonic inductors. Most SCTs are sporadic, without increased risk of aneuploidy if not associated with other anomalies, and are found in fetuses and infants, but SCTs have been reported in adults. The increasing use of prenatal ultrasound exams has dramatically increased the number of fetuses diagnosed with
SCT. Unlike other teratomas, an SCT sometimes grows larger than the fetal body. Fetal SCT, if large enough, predisposes to perinatal complications and even death due to polyhydramnios, premature birth, teratoma rupture, bleeding, fetal hydrops, and labor dystocia. ${ }^{2,3}$ In contrast, postnatal SCT carries a good prognosis with morbidity and mortality determined largely by extent of local disease, the ratio between cystic and solid components, and malignant potential. However, in utero fetal mortality has approached $100 \%$ when SCT is associated with fetal hydrops, which is related to high-output heart

${ }^{1}$ Department of Obstetrics and Gynaecology, Helsinki University Central Hospital, Helsinki, Finland.

Address for correspondence and reprint requests: Vedran Stefanovic, M.D., Ph.D., Department of Obstetrics and Gynaecology, Helsinki University Central Hospital, Haartmaninkatu 2, 00290 Helsinki, Finland (e-mail: vedran.stefanovic@yahoo.com; vedran. stefanovic@hus.fi).

Am J Perinatol Rep 2011;1:87-90. Copyright (C) 2011 by Thieme Medical Publishers, Inc., 333 Seventh Avenue, New York, NY 10001, USA. Tel: +1 (212) 584-4662.

Received: March 5, 2011. Accepted after revision: May 23, 2011. Published online: July 22, 2011.

DOI: http://dx.doi.org/10.1055/s-0031-1284220.

ISSN 2157-6998. 
failure secondary to arteriovenous shunting through the tumor. SCTs, especially if not diagnosed during the pregnancy, can cause severe labor dystocia ${ }^{4}$ with catastrophic consequences to both mother and fetus. In previous decades, the recommended management of SCTs larger than $5 \mathrm{~cm}$ in diameter was delivery by cesarean section to prevent dystocia, tumor rupture, hemorrhage, and fetal death. Rarely reported prenatal ultrasound-guided needle drainage of cystic SCTs offers an alternative to cesarean section that result in decreased risks for both mother and fetus. ${ }^{5,6}$

\section{CASE REPORTS}

\section{Case 1}

A 27-year-old woman with a previous uneventful pregnancy and labor and early first-trimester spontaneous abortion was referred to our tertiary prenatal diagnosis center for level III ultrasound scan at gestational week 19 due to expansion in the fetal sacrococcygeal region. The sonographic evaluation showed a singleton intrauterine pregnancy with normal fetal biometry corresponding to the $19+4$ gestational weeks. In addition, a $27 \times 30 \times 38$-mm tumorous mass arising from the sacrococcygeal region was seen. The mass was predominantly cystic with three smaller lobes and contained some solid tissue without significant vascularization. Otherwise, fetal morphology was normal with an excellent biophysical profile and normal female karyotype. After counseling with the perinatologist, geneticist, and the pediatric surgeon, the parents decided to continue the pregnancy. The fetal growth was appropriate at the regular and frequent checkups with no signs of hemodynamic overload due to the poor vascularization of the teratoma. Magnetic resonance imaging (MRI) was performed at 32 weeks of gestation, which revealed predominantly cystic type I SCT. The size of the teratoma at the last prenatal outpatient visit was $96 \times 72 \times 64 \mathrm{~mm}$. Its circumference was $349 \mathrm{~mm}$ (slightly larger than the abdominal circumference) and the fetus was in a vertex presentation with an excellent biophysical score and the Doppler indices. The cervix was favorable for the induction of labor with Bishop score 5. To avoid possible dystocia during the labor, we decided to drain the cystic parts of the SCT. The fetus was immobilized by administration of $0.35 \mathrm{~mL}$ of vecuronium bromide (Norcuron ${ }^{\circledR}$, Organon, Oss, The Netherlands) into the gluteal region, which was followed by ultrasound-guided removal of 300 $\mathrm{mL}$ of clear fluid with a 22-gauge spinal needle. Successful induction of uncomplicated labor was done by an intravaginal application of a $25-\mu \mathrm{g}$ tablet of misoprostol (Cytotec $^{\circledR}$, Pfizer Aps, Ballerup, Denmark). A 3870-g baby girl was born in excellent condition with Apgar scores of 8 and 10 and umbilical artery $\mathrm{pH}$ of 7.27. A decompressed mass without ulceration was seen in the sacrococcygeal region of the newborn, and the anus and the external genitalia appeared normal and nondisplaced. The mother was discharged home on the third postpartum day. The SCT was removed on the first postnatal day. Tumor markers and histology revealed a mature cystic teratoma. The residual teratoma-tissue was removed laparoscopically 2 months after birth. The infant is now 2 years, 2 months old, and her growth and development are appropriate for her age. The only remnant of the intrauterine process is the unremarkable scar in the presacral area.

\section{Case 2}

A 32-year-old mother of three healthy child was referred for the level III perinatal ultrasound after a secondtrimester screening due to the suspected fetal SCT and two soft markers (intracardiac echogenic focus and short femur).Detailed ultrasound examination showed a single male fetus with a typical type I predominantly cystic SCT with a small and poorly vascularized solid component. Fetal morphology was otherwise normal and the bioprofile was excellent. MRI confirmed cystic type I SCT. After prenatal counseling, an uncomplicated amniocentesis was performed and a normal male karyotype was obtained. The family opted for continuation of the pregnancy, which was followed in the regular outpatient policlinic department. Fetal growth was appropriate and there were no signs of hypervascularization of the SCT. At the last prenatal visit at term, the patient experienced regular spontaneous contractions. The size of SCT was $11 \times 9 \times 17 \mathrm{~cm}$ and estimated volume of $1200 \mathrm{~mL}$. The on-call perinatologist performed an uncomplicated drainage of $950 \mathrm{~mL}$ of clear fluid SCT with a 22-gauge spinal needle in two aspirations with use of the vacuum aspiration, carefully avoiding the solid components of the SCT (Fig. 1). The amniotomy was performed 2 hours later to augment the labor. A 3900-g baby boy was born after the uncomplicated delivery with Apgar scores of 9 and 10 and umbilical artery $\mathrm{pH}$ of 7.28. Decompressed sacrococcygeal mass with the intact covering skin was observed (Fig. 2). The mother was discharged home at the second postpartum day. The SCT was removed on the first postnatal day without complications. The cytological analysis of the removed teratoma fluid was normal with abundant lymphocytes and some macrophages of normal morphology without malignant or dysplastic cells. Recently, at the age of 6 months, the uncomplicated laparoscopic removal of the two small residual presacral cysts in toto was performed. Neurobehavioral development of the infant is uneventful.

\section{DISCUSSION}

One of the most dangerous labor complications in pregnancies complicated by fetal SCT is dystocia due 


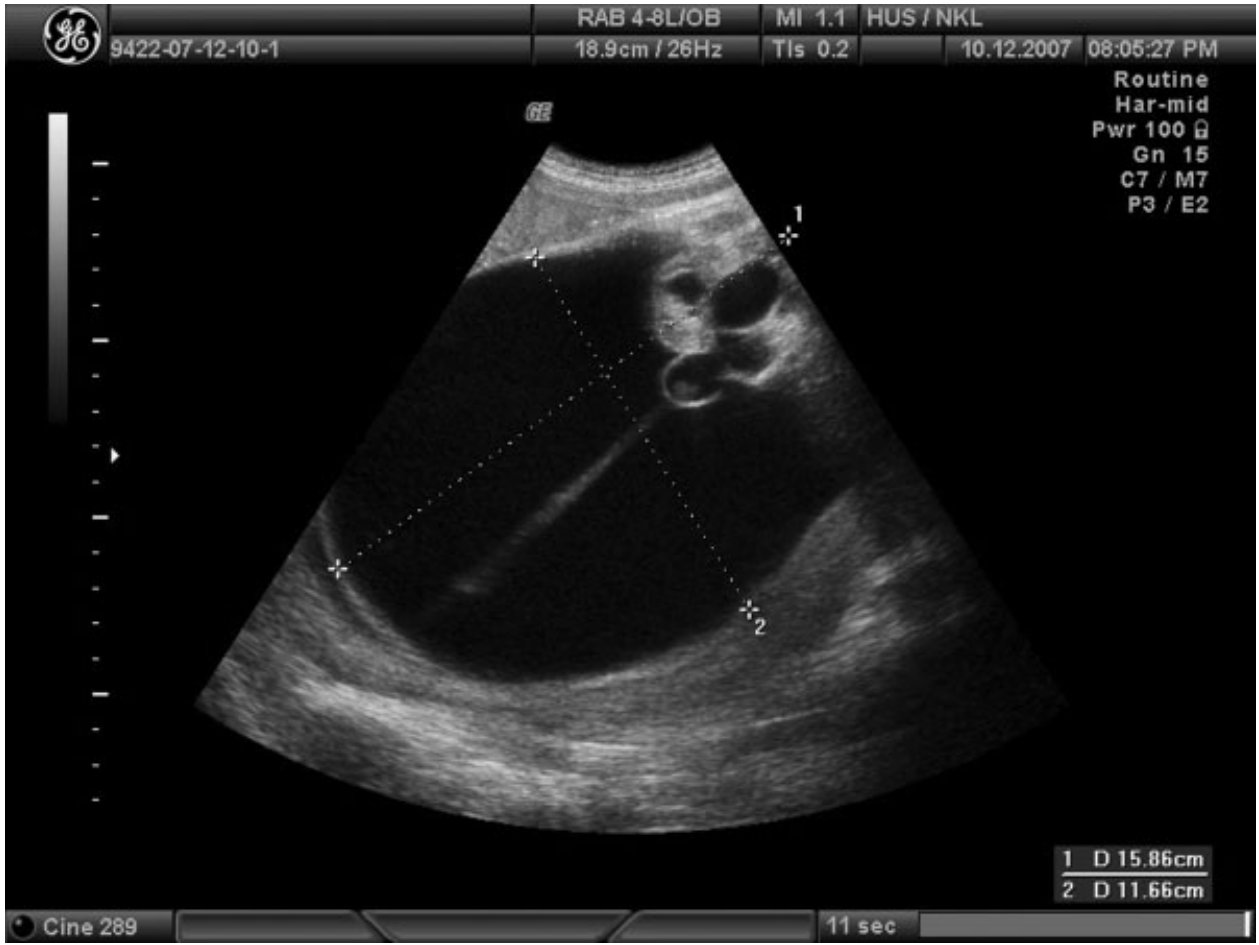

Figure 1 Case 2. The aspiration needle in the cystic teratoma.

to the large size of the tumor, which can lead to the SCT rupture, hemorrhage, and fetal death, as well as the injuries of maternal birth canal. ${ }^{3,4,7}$ With the recent advances in perinatal care, particularly the increasing use of highly sophisticated ultrasound equipment, it is unlikely that unexpected dystocia secondary to this tumor will be seen by many physicians. An ultrasound examination is still the gold standard in diagnosis, and follow-up enables determination of its type and predictions of the outcome. ${ }^{2}$ MRI is the supplementary

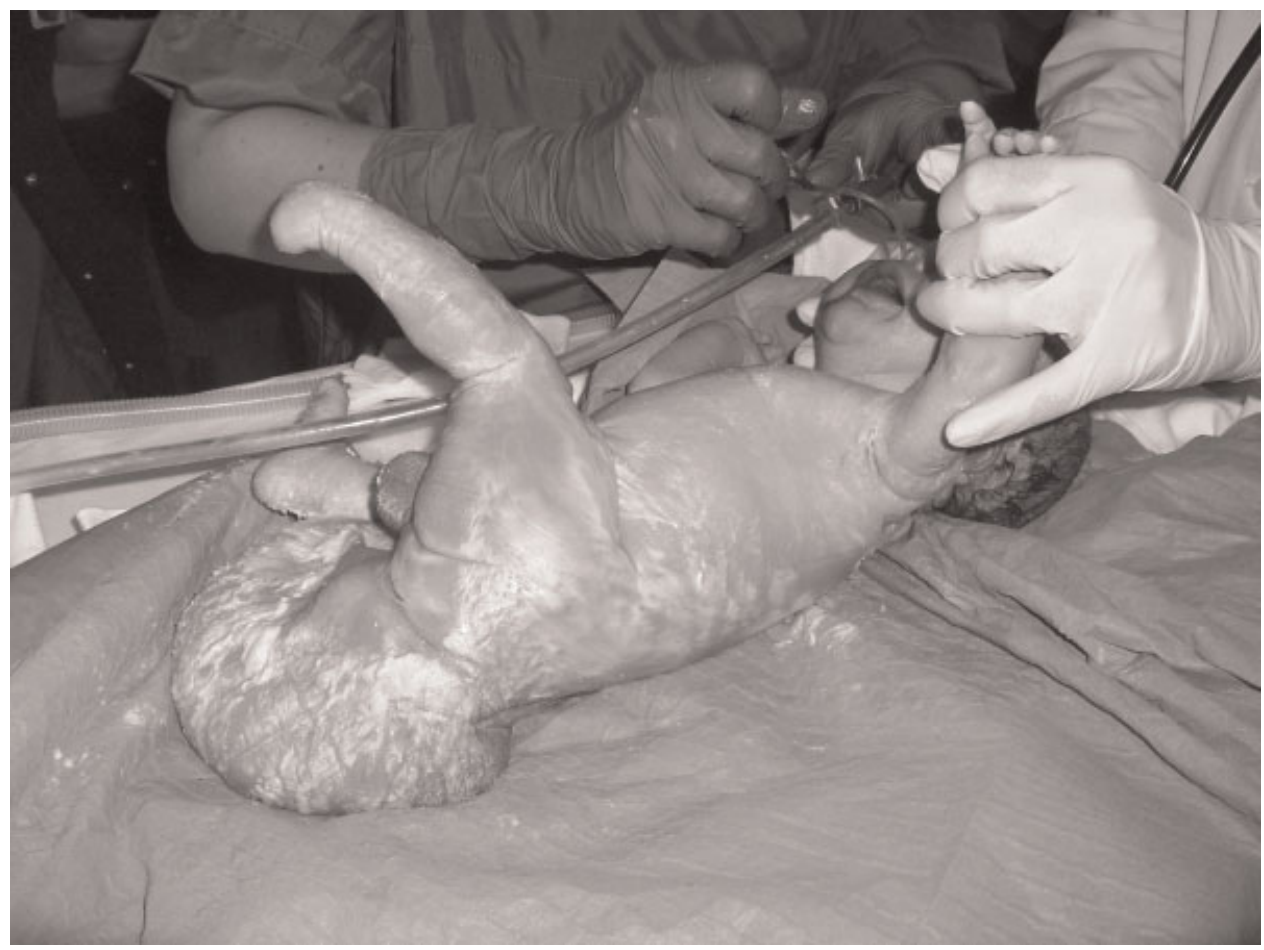

Figure 2 Case 2. Shrunk teratoma immediately after the delivery of the baby boy. Note the normal position of the external genitalia and the anus. 
method useful mostly in solid SCTs with pelvic invasion because MRIs help surgeons better visualize the content and extent of the SCT before performing neonatal surgery. ${ }^{8}$ Most fetuses with giant SCT are delivered by cesarean section. However, there have been a few reported cases of cystic SCT in drainage in term fetuses with subsequent vaginal birth, but only three cases are well documented. ${ }^{5,6}$ We have performed ultrasoundguided needle drainage of cystic SCTs to enable normal vaginal birth, avoid possible dystocia, and prevent possible complications of the cesarean section. The recent study of Patterson et $\mathrm{al}^{9}$ has clearly shown that the maternal morbidity for classic cesarean was significantly increased compared with low transverse cesarean. Additionally, the delivery mode in the potential subsequent pregnancy is usually managed by elective cesarean section due to the increased risk of uterine rupture. Occasionally, spontaneous rupture can happen in nonlaboring pregnant women with a previous vertical incision. Even the elective cesarean section in our two cases would have not eliminated the possibility of SCT rupture, and the need for the extensive vertical incision would have been obvious to deliver fetuses with an intact SCT. We have tried to optimize the time of the teratoma fluid drainage just before the induction of labor when the cervix was favorable for induction or during the onset of spontaneous labor and thus avoid complications of the repeated procedures. Close communication among perinatologists and neonatal surgeons and adequate facilities should be available at any time. Counseling by multidisciplinary approach involving perinatologist, neonatologist, and neonatal surgeon reduces parental anxiety associated with the diagnosis of fetal congenital malformation that is treatable by neonatal surgery and eases decision making about the continuation of pregnancy. ${ }^{10}$

In conclusion, prenatal diagnosis has a major impact on the management of the fetus with SCT. Peripartum ultrasound-guided needle puncture to drain a large cystic teratoma is a safe procedure in experienced hands that minimizes the risks of soft tissue dystocia and allows a normal vaginal delivery, lowering maternal morbidity and the risk of tumor rupture.

\section{ACKNOWLEDGMENT}

We thank Professor Olavi Ylikorkala for his useful suggestions during manuscript preparation.

\section{REFERENCES}

1. Hedrick HL, Flake AW, Crombleholme TM, et al. Sacrococcygeal teratoma: prenatal assessment, fetal intervention, and outcome. J Pediatr Surg 2004;39:430-438; discussion $430-438$

2. Makin EC, Hyett J, Ade-Ajayi N, Patel S, Nicolaides K, Davenport M. Outcome of antenatally diagnosed sacrococcygeal teratomas: single-center experience (1993-2004). J Pediatr Surg 2006;41:388-393

3. Musci MN Jr, Clark MJ, Ayres RE, Finkel MA. Management of dystocia caused by a large sacrococcygeal teratoma. Obstet Gynecol 1983;62(3 Suppl):10s-12s

4. Litwiller MR. Dystocia caused by sacrococcygeal teratoma. Two case reports. Obstet Gynecol 1969;34:783-786

5. Kay S, Khalife S, Laberge JM, Shaw K, Morin L, Flageole H. Prenatal percutaneous needle drainage of cystic sacrococcygeal teratomas. J Pediatr Surg 1999;34:1148-1151

6. Weston MJ, Andrews H. Case report: in-utero aspiration of sacrococcygeal cyst. Clin Radiol 1991;44:119-120

7. el-Shafie M, Naylor D, Schaff E, Conrad M, Miller D. Unexpected dystocia secondary to a fetal sacrococcygeal teratoma: a successful outcome. Int J Gynaecol Obstet 1988;27:431-438

8. Kemp J, Davenport M, Pernet A. Antenatally diagnosed surgical anomalies: the psychological effect of parental antenatal counseling. J Pediatr Surg 1998;33:1376-1379

9. Patterson LS, O'Connell CM, Baskett TF. Maternal and perinatal morbidity associated with classic and inverted $\mathrm{T}$ cesarean incisions. Obstet Gynecol 2002;100:633-637

10. Crombleholme TM, D'Alton M, Cendron M, et al. Prenatal diagnosis and the pediatric surgeon: the impact of prenatal consultation on perinatal management. J Pediatr Surg 1996;31:156-162; discussion 162-163 